\section{VERS LA CONCLUSION DE L'AFFAIRE GALLO-POPOVIC?}

Les conditions dans lesquelles a été obtenue, pour la première fois, une culture continue du virus HIV dans le laboratoire du Professeur Gallo ont fait I'objet d'une longue enquête de I'OSI loffice of scientific integrity) du NIH. Au-delà des remous qui agitent actuellement cette institution du NIH et des critiques faites de toutes parts sur le style de son rapport final, certaines conclusions importantes émergent. II faut remarquer que cette émergence, largement médiatisée, survient alors que le rapport aurait dû rester encore secret pour plusieurs mois. Ce secret est, néanmoins, tout relatif et le numéro du 16 août 1991 de Science (vol. 253, pages 728-32) consacre une longue analyse à ce document ainsi qu'aux réactions des chercheurs mis en cause. Les principales critiques sont faites à Mikulas Popovic, un scientifique tchèque qui travaillait à cette époque dans le laboratoire de Robert Gallo, au NCl (National Cancer Institute) de Bethesda [1]. L'article rapportant la découverte du virus, appelé à l'époque HTLV III, et sa culture continue, indiquait que cette culture durait depuis 5 mois. En réalité, elle n'était vieille que de 4 mois lors de la réception du manuscrit... et n'était pas continue puisque Popovic y rajoutait du virus frais lorsque le pourcentage de cellules infectées tombait en dessous de 10 à $30 \%$.

Cette culture, faisant suite à plusieurs essais infructueux, avait été initiée grâce à un mélange de dix isolats viraux différents que la publication de 1984 disait avoir tous été testés pour la présence d'une activité de transcriptase inverse, indication d'une réplication virale active. En réalité, ce test n'était positif que pour un seul des isolats analysés. De plus, il n'est pas exact d'indiquer que cette culture continue était la première à être réussie dans le laboratoire de Robert Gallo : I'échantillon du virus LAV, envoyé par l'équipe du Professeur Luc Montagnier (Institut Pasteur de Paris) avait auparavant été mis en culture, avec succès. Cette culture attendait au réfrigérateur. On sait que le virus HTLV III décrit dans la publication de Gallo était, en réalité, un contaminant issu de l'échantillon adressé de Paris.

Enfin, un tableau, toujours dans cet article de 1984, présente des résultats "ND " (not done). En fait, ces résultats d'étude en immunofluorescence de clones cellulaires infectés par HTLV III avaient été obtenus... mais étaient négatifs.

Enfin, il est reproché à Popovic de n'avoir pas comparé HTLV III au virus LAV précédemment décrit par l'équipe de I'Institut Pasteur [2]. II s'avère que le manuscrit contenait initialement une telle discussion, qui a été supprimée à la demande de Robert Gallo qui dit avoir désiré, à l'époque, la reprendre dans un article co-signé avec les chercheurs de l'équipe française.

En résumé, il est maintenant établi que le virus HIV 1 a initialement été détecté et isolé par l'équipe de Montagnier. En réalité, du fait d'une contamination survenue à I'Institut Pasteur, ce sont deux virus différents qui ont été, sans le savoir, le premier mis en évidence (article de Science en 1983 [2]), le second cultivé et caractérisé par la séquence de son génome $[3,4]$.

Ce deuxième virus, étudié à l'Institut Pasteur, a été, dès 1983, cultivé avec succès dans le laboratoire du Professeur Gallo. Une autre culture, initiée dans le même laboratoire, $a$, en fait, été contaminée par ce même virus qui a, par conséquent, été étudiée, à nouveau et séparément, par les chercheurs du $\mathrm{NCl}$. La première publication de leurs résultats par les chercheurs de cette équipe souffre de certaines imprécisions et incorrections masquant, en partie, les conditions dans lesquelles ils avaient été obtenus.

A.K.

[1. Popovic M, Sarngadharan MG, Read E, Gallo RC. Science 1984 ; 224 : 497-500.]

[2. Barré-Sinoussi $F$, et al. Science $1983 ; 220: 868-70$.

[3. Alison $M$, et al. Nature 1984; 312 : 757-60.]

[4. Weil-Hobson S, Sonigo P, Danos O, Khol S, Alison M. Cell $1985 ; 40$ : 9-17.].

\section{BRÈVES}

Un transporteur des acides aminés basiques est le récepteur d'un rétrovirus murin. Les virus utilisent comme récepteurs des protéines membranaires qui sont, de ce fait, détournées de leur fonction physiologique. C'est ainsi que le virus du SIDA, HIV, a comme récepteur la molécule CD4 alors que le virus d'Epstein-Barr utilise l'antigène de surface CR2 (un récepteur d'une fraction du complément), les rhinovirus, la molécule d'adhérence intercellulaire ICAM-1 et, encore que cela soit plus discuté (voir brève de ce numéro, p. 862), le virus Herpès utilise des récepteurs du FGF (fibroblast growth factor) [1]. Le récepteur du rétrovirus écotropique de souris MuLV (murine leukemia virus) a vu son ADN complémentaire cloné en 1989 par l'équipe de Cunningham, de Boston (MA, USA) [2]. Il s'agit d'une protéine de 622 acides aminés comportant 14 domaines transmembranaires potentiels. L'équipe de Cunningham [3] ainsi qu'une équipe de Portland (OR, USA) [4] viennent maintenant d'injecter des ARN codant pour ce récepteur dans les œufs de Xénope. Les deux équipes montrent qu'elles peuvent alors induire un transport accéléré d'acides aminés basiques vers le milieu intracellulaire. Associé au fait que le gène de mammifère présente de nettes analogies avec les gènes de levure codant pour des transporteurs d'acides aminés, cela suggère que le récepteur de MuLV est bien un transporteur d'acides aminés basiques.

Plus récemment, une autre équipe rapportait, dans un congrès, le clonage du récepteur d'un virus leucémogène du gibbon, le virus GALV gibbon ape leukemia virus [cité dans la référence 5]. Cette dernière molécule ressemble étrangement au transporteur de phosphate du champignon Neurospora crassa. Il n'est pas du tout certain que le type de récepteur utilisé par les différents rétrovirus ait toujours une signification pathogénique. Cependant, l'interaction du virus de Friend avec le récepteur de l'érythropoïétine évoque un évident $\mathrm{m} / \mathrm{s} n^{\circ} 8$, vol. 7 , octobre 91 


\section{aI BRÈVES aII}

mécanisme d'induction de l'hyperplasie des cellules érythroïdes provoquée par ce virus $\left(\mathrm{m} / \mathrm{s}, n^{\circ} 4\right.$, vol. 6 , p. 403). Dans d'autres cas, le virus ne fait, probablement, qu'exploiter la présence d'une protéine cellulaire de surface pour infecter la cellule, la totalité des conséquences pathologiques de cette infection étant liée à des phases postérieures à la liaison du virus à son récepteur.

[1. Courtois Y, médecine/sciences 1990 ; 7: 674-9.]

[2. Abritton LM, et al. Cell 1989 ; 57 : 659-66.]

[3. Kim JW, et al. Nature 1991; 352: 725-8.]

[4.Wang H, et al. Nature $1991 ; 352$ : 729-31.]

[5.Vile RG, Weiss RA. Nature 1991 ; $352: 666-7$.

Granulomatose chronique, NADPH oxydase et petite protéine G Krev-1. La granulomatose chronique est caractérisée par un déficit des polynucléaires neutrophiles en la production de l'anion supéroxyde $\mathrm{O}_{2}{ }^{-}$, étape essentielle à l'activité bactéricide de ces leucocytes. L'anomalie réside au niveau d'un système enzymatique membranaire producteur de $\mathrm{O}_{2}{ }^{-}$, la NADPH oxydase, dont la sous-unité de $90 \mathrm{kDa}$, codée par un gène situé sur le chromosome $\mathrm{X}$, est la plus souvent en cause dans la maladie $\left(\mathrm{m} / \mathrm{s}, n^{\circ} 9\right.$, vol. 2, p. 521 ; $n^{\circ} 2$, vol. 5, p. $126 ; n^{\circ} 6$, vol. 7, p. 627). Cette NADPH oxydase peut être stimulée en traitant des polynucléaires entiers par différents agents mimant un contact avec un agent infectieux qui se lie à la membrane et est phagocyté. Cette activation est bloquée par la toxine de Bordetella pertussis, indiquant qu'elle nécessite le relais d'une grande protéine $G$ trimérique de type $\alpha \beta \gamma$. En revanche, un extrait acellulaire de polynucléaires n'a aucune activité NADPH oxydase, à moins qu'il ne soit stimulé par l'acide arachidonique. Cette stimulation est insensible à la toxine de Bordetella pertussis, mais semble également se faire par l'intermédiaire d'une protéine G. Différentes approches biochimiques et immunologiques identifient cette protéine $G$ à un membre de la famille p2 $1^{\text {ras }}$ qui semble immunologiquement similaire à Krev-1. Il a été montré, en 1989, que cette p21 Krev-1 pouvait se comporter comme une substance antiproliférative, inversant la transformation phénotypique induite par l'oncogène ras activé $\left(\mathrm{m} / \mathrm{s}, n{ }^{\circ} 4\right.$, vol 5 , p. 260). Plus récemment, Krev-1, aussi appelé Rap-1, a été localiséc dans le complexe de Golgi, alors que p21 Hras et p21 Kiras sont localisées à la face interne de la membrane plasmique $\left(\mathrm{m} / \mathrm{s}, n^{\circ} 5\right.$, vol. 7, p. 518). Les équipes américaines de l'Indiana et de Pennsylvanie [1] décrivant le rôle de Krev-1 dans l'activation de la NADPH oxydase, probablement en aval de la mise en jeu de la grande protéine $G$ trimérique, détectent Krev-1 dans la fraction cytosolique, mais soulignent qu'il peut fort bien s'agir là d'un artefact de préparation. Nous nous trouvons donc là devant une nouvelle fonction pour ces très nombreux membres de la famille des petites protéines $G$ qui peuvent intervenir dans la régulation de la prolifération cellulaire, le transit intracellulaire et l'ensemble des processus secrétoires, et maintenant l'activation d'une enzyme essentielle à la fonction bactéricide et cytotoxique des polynucléaires neutrophiles. [1.Eklund EA, et al. J Biol Chem
1991; $266: 13964-70$.

Des anticorps synthétiques non protéiques La spécificité idiotypique des anticorps est due à six boucles dénommées CDR (complementary determining regions) situées dans les régions hypervariables des parties variables des sous-unités de molécules d'immunoglobulines. Des peptides reproduisant la séquence de ces boucles peuvent avoir la même spécificité idiotypique, c'est-à-dire reconnaître exactement les mêmes cibles avec une bonne affinité. C'est ainsi qu'a été fabriqué un peptide reproduisant le motif spécifique d'une boucle
CDR d'un anticorps dirigé contre le récepteur des rhéovirus. Les points de contact entre cette molécule et sa cible ont été analysés en détail et une molécule organique reproduisant la structure d'ensemble de ce peptide a été synthétisée. Contrairement au peptide dont elle est l'image, cette molécule, dénommée mimetic est hydrosoluble, résistante aux protéases, et probablement diffusible à travers la barrière hémato-encéphalique. Les équipes américaines de Philadelphie (PA) et de Chicago (IL) ayant réalisé ce travail ont démontré l'affinité de ce mimetic pour les mêmes cibles que l'anticorps, notamment pour le récepteur des rhéovirus [1]. En effet, au même titre que l'anticorps entier, le mimetic interagissant avec le récepteur des rhéovirus de cellules $\mathrm{T}$ bloque leur prolifération induite par un traitement par la concanavaline A. La mise en évidence, notamment grâce aux méthodes du clonage moléculaire, d'un nombre croissant de ligands et de récepteurs, cibles potentielles d'une action pharmacologique, conduit à imaginer une grande diversité de modulateurs, agonistes ou antagonistes, le plus souvent de nature peptidique. Ces peptides sont, cependant de faible intérêt pour une utilisation thérapeutique. Ils ne peuvent être, le plus souvent, administrés par voie orale, sont instables et souvent compartimentés dans des secteurs où ils ne sont pas actifs. La conception assistée par ordinateur de médicaments (drug design) permet, notamment, de modéliser des molécules non peptidiques mais de conformations équivalentes et qui pourraient ainsi avoir les mêmes propriétés biologiques. L'éventail des possibles utilisations de telles molécules est immense, faisant tout l'intérêt de ces premiers résultats démontrant l'identité idiotypique entre un fragment peptidique d'anticorps et une molécule organique non peptidique.

[1. Saragovi HU, et al. Science 1991 253: 792-5.] 


\section{an BRÈVES ant}

Confirmation que CFTR est bien un canal chlore sensible à l'AMP cyclique. Le CFTR (cystic fibrosis gene encoded transmembrane regulator) est le produit du gène CF de la mucoviscidose. La perte de fonction, dans cette maladie, d'un canal chlore normalement stimulé par l'AMP cyclique dans les cellules des glandes sudoripares et de l'épithélium bronchique avait suggéré que le gène CF codait pour un composant ou un régulateur de ce canal chlore. A l'heure actuelle, le consensus semble se faire sur l'identité du CFTR qui serait, par lui-même, tout ou partie du canal altéré dans la mucoviscidose. M. P. Anderson et al., de l'université de l'Iowa (Iowa City, IA, USA), associés à des équipes du Massachusetts, viennent de montrer qu'une mutation dirigée de l'ADN complémentaire du CFTR, convertissant les lysines 95 et 335 en acides aminés acides, modifiait la sélectivité du canal chlore dans des cellules infectées par un rétrovirus recombinant contenant la séquence CFTR. Alors que cette sélectivité va normalement dans le sens d'une plus grande perméabilité pour les anions bromures, suivis des chlorures, des iodures et des fluorures, la mutation des résidus lysines entraîne une sélectivité accrue pour les iodures, alors que la perméabilité aux bromures, aux chlorures et aux fluorures est inférieure [1]. D. B. Rich et al., des mêmes laboratoires que précédemment, viennent de compléter cette étude en montrant que la délétion d'une région spécifique du CFTR modifiait sa susceptibilité à l'AMP cyclique. La protéine est normalement constituée de deux parties similaires, chacune d'entre elles comportant un domaine trans-membranaire et un domaine de fixation présomptive des nucléotides (NBD, nucleotide binding domain). Ces deux parties de la molécule sont séparées par une région particulière dénommée $R$, au niveau de laquelle existent plusieurs sites potentiels de phosphorylation par les protéines kinases dépendan- de $\mathrm{R}$ aboutit à la synthèse d'un canal qui est actif en permanence, ne nécessitant donc pas d'activation préalable par l'AMP cyclique. Cependant, cette activité peut encore être stimulée par l'AMP cyclique, indiquant que d'autres sites de réponse aux protéines kinases stimulées par ce second messager existent en dehors du domaine $\mathrm{R}$. Les auteurs montrent également que la délétion de $\mathrm{R}$ permet de restaurer la perméabilité du canal chlore, portant une mutation au niveau du domaine NBD2, mais non au niveau du domaine NBD1. Il semble donc y avoir une interaction spécifique entre $\mathrm{R}$ et la région de fixation des nucléotides NBD2 [2].

[1. Anderson MB, et al. Science 1991 ; 253 : 202-5.]

[2. Rich DP, et al. Science 1991 ; 253 : 205-7.]

Efficacité in vivo de l'immunoadhésine CD4. La protéine de surface CD4, agissant normalement comme une molécule d'adhérence des lymphocytes $\mathrm{T}$, est également le récepteur principal du virus HIV-1. La stratégie de lutte contre l'infection par HIV-1 grâce à l'administration de leurres moléculaires a été explorée depuis plusieurs années. La molécule CD4 recombinante n'est pas propice à cette utilisation car sa demi-vie dans la circulation est extrêmement faible. C'est pour stabiliser cette molécule que la société Genentech (San Francisco, CA, USA) a synthétisé par génie génétique une molécule hybride entre le fragment Fc de l'immunoglobuline humaine IgG1 du côté C-terminal et deux régions de type immunoglobuline de CD4 du côté $\mathrm{N}$-terminal. Du fait de la stabilisation conférée par le fragment Fc, la concentration de l'immunoadhésine CD4 est 25 fois plus importante que celle de la protéine CD4 recombinante administrée à la même dose. Restait à démontrer que cette molécule était active in vivo. Pour ce faire, R. H. R. Ward et al., chercheurs de plusieurs laboratoires américains, ont traité des chimpanzés par l'immunoadhésine CD4 immédiatement avant une infection intraveineuse par une préparation virale HIV-1 IIIB (une lignée virale maintenue sur culture cellulaire), puis pendant plusieurs semaines [1]. Cette souche IIIB de HIV-1 n'est pas pathogène pour le chimpanzé mais provoque normalement une virémie 4 à 6 semaines après l'inoculation. Le traitement par l'immunoadhésine permet de prévenir cette virémie. Ces résultats sont intéressants en ce qu'ils démontrent, pour la première fois, une efficacité in vivo d'un leurre moléculaire. Les difficultés restent cependant considérables. Les doses administrées dans l'expérience rapportée ci-dessus sont énormes (environ six grammes d'immunoadhésine... c'est-à-dire une petite fortune). Les résultats sont obtenus avec la souche IIIB, dont il est possible qu'elle ait perdu beaucoup de sa virulence en culture ex vivo. Enfin, on peut se demander s'il n'est pas dangereux d'injecter un leurre moléculaire capable de fixer HIV-1 et contenant un fragment Fc d'immunoglobuline. Il a, en effet, été démontré que l'infection par HIV-1 de cellules non lymphocytaires, par exemple de macrophages, pouvait se faire par internalisation d'un complexe antigène-anticorps, via la fixation sur le récepteur, du fragment Fc. En revanche, cette immunoadhésine a l'avantage de pouvoir, comme les immunoglobulines, franchir la barrière placentaire. L'on pourrait par conséquent imaginer de l'utiliser en période prénatale afin d'éviter l'infection des nouveau-nés, infection qui semble se produire principalement durant l'accouchement.

[1. Ward RHR, et al. Nature 1991 ; 352 : 434-6.]

Les fibrillines, une famille de protéines altérées dans le syndrome de Marfan et les maladies apparentées. La localisation sur le chromosome 15 du gène du syndrome de Marfan, prototype des 
affections du tissu conjonctif, a été annoncée en $1990\left(\mathrm{~m} / \mathrm{s} n^{\circ} 10\right.$, vol. 6 , p. 1018), et un gène candidat désigné, celui d'une glycoprotéine, la fibrilline, dont la synthèse est altérée dans les cellules en culture de ces malades. Confirmation et précisions sont apportées par un bouquet d'articles parus dans Nature. Le gène de la fibrilline, localisé avec précision grâce à l'hybridation in situ, est en 15q21; il a été cloné et séquencé par deux groupes [1, 2] ; les zones séquencées par les deux équipes se chevauchant partiellement, elles identifient en tout environ 2000 acides aminés, ce qui devrait couvrir la totalité de la séquence; la taille du messager est de $10 \mathrm{~kb}$, et celle de la protéine de $350000 \mathrm{Da}$.

Deux articles démontrent une liaison génétique entre fibrilline et syndrome de Marfan. Grâce à des marqueurs polymorphiques, cette liaison a été trouvée par Lee et al. (dans une collaboration internationale, USA, Japon, France) [1] avec un lod score de 7,56, et par Dietz et al. (Baltimore, MD et Portland, OR, USA) [3] de 3,9. De plus [3], chez deux malades non apparentés, une même mutation Arg $\rightarrow$ Pro au codon 239*, apparue de novo car non présente chez les parents, a été découverte. Il convient toutefois de noter que c'est dans deux cas seulement sur 33 testés qu'une mutation a été observée jusqu'à présent. Chez aucun malade l'ADN n'a révélé de délétion ni de remaniement visible.

Lee et al. [1] ont fait une autre importante découverte, lorsqu'ils ont observé qu'à faible stringence, une sonde d'ADNc de fibrilline s'hybride avec des clones génomiques provenant du chromosome 5 . Une séquence appelée Fib 5 a été obtenue ; les ressemblances entre les deux protéines étant surtout constituées par des répétitions de séquences du type EGF. Les auteurs ont complété ce travail en cherchant une liaison génétique entre la Fib 5 et des syndromes voisins de celui de Marfan. Dans deux familles atteintes d'une maladie à transmission domi- nante nommée arachnodactylie congénitale contracturante, ils ont trouvé une liaison avec un lod score de 4,5. Enfin, une troisième série de clones, en cours d'étude, semble provenir d'un troisième gène de fibrilline situé sur le chromosome 17. Ainsi se dessine une nouvelle famille de protéines, les fibrillines, et de maladies qui semblent dues à leur altération, et dont l'hétérogénéité nouvellement reconnue les fera peut-être marcher sur les traces du collagène. Signalons enfin un fait anecdotique, mais que beaucoup de sujets atteints d'anomalies héréditaires accueillent avec faveur : il semble que plusieurs personnages célèbres aient présenté, ou pu présenter, un syndrome de Marfan. Le plus illustre est sans doute Abraham Lincoln, dont les restes, conservés dans un musée de Washington, vont bientôt être soumis à la miraculeuse réaction d'amplification de l'ADN pour leur faire subir un interrogatoire sur leurs gènes de fibrilline [4].

[1. Lee B, et al. Nature 1991; 352 : 330-4.]

[2. Maslen CL. Nature 1991 ; 352 : 334-7.]

[3. Dietz HC, et al. Nature 1991 ; 352 : 337-9.]

[4. McKusick VA. Nature 1991 ; 352 : 279-81.]

\section{- Nomenclature selon [2].}

Quand les abzymes deviennent de vraies enzymes. Les lecteurs de médecine/sciences savent que les abzymes sont des anticorps qui reconnaissent un état de transition d'une molécule et qui catalysent ainsi la réaction nécessitant que l'un des réactants passe 'par cet état. L'extraordinaire diversité des anticorps possibles et la possibilité d'augmenter encore cette diversité grâce à la production d'anticorps dans $E$. Coli après recombinaison dans le phage $\lambda$ $\left(\mathrm{m} / \mathrm{s} n^{\circ} 1\right.$, vol. 6, p. 82) font des abzymes des catalyseurs potentiellement universels qui pourraient être des réactifs d'avenir, aussi bien en chimie qu'en biologie et en médecine. Cependant, l'activité catalytique des premières abzymes était relativement faible comparée à celle des enzymes. Des résultats plus récents montrent que l'activité des abzymes ne se limite pas, en fait, à la facilitation d'une réaction via la stabilisation d'un état de transition : une fois l'espèce transitionnelle fixée à l'anticorps, des domaines et des résidus de l'immunoglobuline peuvent intervenir directement dans le processus catalytique, selon des mécanismes mimant étroitement le mode d'action des enzymes. Le groupe de R. A. Lerner (La Jolla, CA, USA) vient ainsi de montrer qu'un anticorps monoclonal dirigé contre un analogue d'un état de transition d'une réaction de transestérification catalysait une telle réaction selon un mécanisme enzymatique connu sous le nom de "pingpong ". Ce mécanisme comporte la formation de complexes ternaires liés de façon covalente entre la protéine enzymatique et des intermédiaires de réaction. Un premier substrat se fixe à l'enzyme, puis un premier produit est libéré. Le second substrat se fixe à son tour à l'enzyme modifiée de façon covalente et le second produit est enfin libéré alors que l'enzyme est régénérée [1]. D'autres exemples de ce type démontrant la nature enzymatique des réactions catalysées par les abzymes ont été rapportés par d'autres auteurs cités par R. A. Lerner et al. [2]. Il semble ainsi que les anticorps se comportent non seulement comme des récepteurs spécifiques d'un état intermédiaire d'un réactant, mais aussi comme des protéines chimiquement réactives. Des modifications dirigées de l'anticorps, l'addition à son niveau de groupes chimiques particuliers pourraient encore stimuler considérablement l'efficacité de cette réactivité catalytique. L'espoir existe donc aujourd'hui d'avoir là un moyen de fabriquer, presque à volonté, une nouvelle classe de catalyseurs biologiques aussi efficaces que les enzymes.

[1. Wirsching $\mathrm{P}$, et al. Science 1991 ; 252: 680-5.]

[2. Lerner RA, et al. Science 1991 ; 252: 659-67.] 
- Réponse immune de l'intestin humain à une vaccination orale. L'étude de la réponse immune locale de l'intestin, in vivo chez l'homme, à une stimulation antigénique orale est toujours difficile, et les données physiologiques ou physio-pathologiques restent très fragmentaires. L'étude des coproanticorps n'a pas répondu à tous les espoirs fondés en eux. Le travail de Marianne Quiding et al. [1] (département de microbiologie et d'immunologie, Université de Göteborg, Suède) est d'un intérêt tout particulier, puisqu'il étudie la réponse lymphoïde de la muqueuse intestinale chez l'homme à une vaccination anticholérique orale $\left(10^{11} \mathrm{~V}\right.$. cholerae et $1 \mathrm{mg}$ de la sous-unité $B$ de la toxine cholérique $[\mathrm{B}-\mathrm{TC}])$, et la compare à la réponse systémique, grâce à une technique ELISPOT [2] (enzyme-linked immunospot assay) appliquée aux cellules $\mathrm{B}$ et $\mathrm{T}$, notamment à celles de la muqueuse duodénale, isolées à partir de biopsies endoscopiques par une nouvelle technique de dispersion enzymatique [3]. La vaccination orale provoque, au bout de huit jours, une augmentation significative du nombre de cellules sécrétrices d'anticorps (CSA) dans le duodénum, avec notamment apparition de nombreuses CSA dirigées contre (B-TC). Une deuxième immunisation orale, 15 jours après la première, induit une réponse locale plus marquée que la première, dominée par des CSA antiB-TC de classe essentiellement IgA, puis $\operatorname{Ig} G$; la réponse de classe IgM ne concerne qu'un petit nombre de cellules. Une immunisation de rappel cinq mois plus tard induit une réponse anticorps très intense chez tous les sujets étudiés, comparable à celle obtenue après la deuxième immunisation, tant en intensité qu'en distribution isotypique des CSA. La réponse systémique, évaluée dans le sang périphérique, comporte des CSA anti B - TC de classe IgA et IgG en proportion similaire : elle est de moins grande intensité qu'à l'échelon intestinal et de brève durée, les CSA disparaissant en quelques jours. La réponse systémique à l'immunisation vée lors de la deuxième dose. Particulière à la muqueuse duodénale est la réponse concernant les cellules sécrétant l'interféron $\gamma(\mathrm{C}-\mathrm{IFN}-\gamma)$ : déjà nombreuses avant immunisation dans la muqueuse duodénale, les CIFN- $\gamma$ augmentent significativement en densité, surtout après la deuxième immunisation, sans modification de leur nombre en périphérie. Ce travail indique qu'il est maintenant possible d'étudier, à l'échelon cellulaire, la réponse immune intestinale in vivo chez l'homme : il confirme les différences marquées entre la réponse locale et périphérique après immunisation orale et confirme aussi la notion de mémoire immunologique de la muqueuse intestinale. Le fait, établi par ce travail, que l'intestin est une source importante de production d'IFN- $\gamma$ est particulièrement intéressant à la lumière des récents travaux suggérant le rôle de celui-ci dans la perméabilité et la fonction barrière de cet organe.

[1. Quiding $\mathrm{M}$, et al. J Clin Invest 1991; 88 : 143-8.]

[2. Czerkinsky C, et al. J Immunol Methods 1988 ; 115 : 31-7.]

[3. Nordström I, et al. Advances in mucosal immunology. Mcdonald TT, et al. eds Dordrecht, Kluwer, 1990 : 103-4.]

Canal chlore et acidification intracellulaire : mécanisme des anomalies de sécrétion bronchique dans la mucoviscidose. Des arguments de plus en plus convaincants indiquent que le gène de la mucoviscidose, codant pour le CFTR (cystic fibrosis gene encoded transmembrane regulator, $\mathrm{m} / \mathrm{s} n^{\circ} 8$, vol. 5, p. 589), contrôle directement l'activité d'un canal chlore sensible à l' AMP cyclique (voir brève p. 872, ce numéro). Le CFTR pourrait être lui-même ce canal chlore ou, du moins, l'une de ses sous-unités. L'absence de réabsorption du chlore explique, probablement, l'insuffisance pancréatique et l'ileus méconial dus à des obstructions en rapport avec l'hyperviscosité des sécrétions pancréatiques et du méconium. Ce sont, cependant, les complications bronchiques qui font toute la gravité de la mucoviscidose : surinfections à répétition, destruction bronchique, dilatation des bronches, etc. [1]. Une des importantes modifications qualitatives des sécrétions bronchiques des malades atteints de mucoviscidose est une diminution de la sialylation et une augmentation de la sulfatation et de la fucosylation des glycoprotéines. J. Barasch, de l'université Columbia à New York (USA), [2] vient de montrer un déficit généralisé de l'acidification des vésicules intracellulaires (trans-Golgi, prélysosomes, endosomes) dans les cellules de malades atteints de mucoviscidose. Il semble que le $\mathrm{pH}$ intracellulaire de ces vésicules soit réglé par une pompe à protons et par le canal chlore. Le fonctionnement de la pompe à protons serait constitutif, le $\mathrm{pH}$ intracellulaire étant contrôlé par l'ouverture du canal chlore. Chez les mucoviscidosiques, l'inactivation de ce canal chlore s'accompagne donc d'une alcalinisation des vésicules intracellulaires de nature à modifier l'activité de leurs enzymes. La sialyl-transférase est ainsi particulièrement sensible au $\mathrm{pH}$, son maximum d'activité étant observé à $\mathrm{pH}$ acide. Une diminution de l'activité de cette sialyl-transférase entraînerait une augmentation de l'activité des enzymes responsables de la fucosylation et de la sulfatation qui sont, quant à elles, actives à $\mathrm{pH}$ neutre et fonctionnent compétitivement avec la sialyl-transférase. La modification de la nature des glycoprotéines, l'apparition dans les sécrétions bronchiques d'une concentration anormale d'asialoglycoprotéines, pourraient être également impliquées dans la particulière susceptibilité à l'infection par Pseudomonas qui pourrait adhérer spécifiquement aux parois bronchiques sécrétant ces protéines désialées.

[1. Pennacino-Sauvage M, Hulen C. médecine/sciences $1990 ; 6$ : 886-94.]

[2. Barasch J, et al. Nature 1991 352 : 70-3.]

Un ARN et une protéine antiprion? On sait que le gène des prions contient une phase ouverte de lecture insérée dans un seul long exon. Goldgaber (New York) a 


\section{口 BRÈVES}

analysé par ordinateur [1] la séquence complémentaire de cet exon et a constaté qu'elle pouvait présenter une phase ouverte, permettant de déduire une séquence protéique unique, dont le diagramme d'hydropathie est une image en miroir de celui du prion $(\operatorname{PrP})$, et qu'il propose d'appeler antiPrP. Dans cette séquence, on peut noter que la $1^{\text {re }}$ base d'un codon sur un brin correspond à la $3^{\mathrm{e}}$ du codon de l'autre brin, ce qui doit contribuer à la conservation du gène au cours de l'évolution. Goldgaber fait également remarquer que les mutations connues associées à une maladie de Gerstmann-Sträussler (codons 102 et 117) doivent provoquer un changement d'acide aminé sur l'anti-PrP, mais non celles qui sont associées à une maladie de Creutzfeldt-Jakob (codons 178 et 200). On peut vérifier que le polymorphisme en position 129, dont l'importance a peut-être été sous-estimée ( $\mathrm{m} / \mathrm{s} n^{\circ} 7$, vol. $\left.7, p .754\right)$ n'aurait pas non plus d'influence sur l'anti-PrP : en effet AUG (Met) $\rightarrow$ GUG (Val) sur le $\operatorname{PrP}$, alors que CAU et CAC sur l'anti-PrP codent tous deux pour une His.

L'auteur suggère donc de rechercher le transcrit et la protéine correspondant au gène anti-PrP. On conçoit qu'un tel défi ne soit pas resté longtemps sans être relevé. Manson et Hope (Edimbourg) ont tenté [2] une hybridation in situ avec des sondes d'ARN marqué au ${ }^{35} \mathrm{~S}$ dans le cerveau de souris infectées ou non par la tremblante. Les résultats sont restés négatifs pour l'ARN anti-PrP. En revanche, Hewinson et al. (Weybridge, Surrey, GB) ont réussi [3], chez le veau, à mettre en évidence une hybridation entre l'ARN cellulaire total de plusieurs tissus et un ARN simple brin spécifique de $\operatorname{PrP}$ comme d'anti-PrP, l'activité anti-PrP semblant être environ 10 fois plus faible que l'autre. Si la protéine anti$\operatorname{Pr} P$ est effectivement synthétisée, il est possible qu'une interaction s'exerce entre les deux protéines et qu'une altération de cette interaction contribue à la genèse des encéphalopathies spongiformes.

$\mathrm{m} / \mathrm{s} n^{\circ} 8$, vol. 7 , octobre 91
[1. Goldgaber D. Nature 1991 ; 351 : 106.]

[2. Manson J, Hope J. Nature 1991 ; 352: 291.]

[3. Hewinson RG, et al. Nature $1991 ; 352$ : 291.]

$\square$ Régulation post-traductionnelle du facteur de transcription Pit 1. Le facteur de transcription Pit 1 appartient à la famille des protéines se fixant à l'ADN grâce à un domaine peptidique homéo et Pou $\left(\mathrm{m} / \mathrm{s}, n^{\circ} 3\right.$, vol. 5, p. 172). Pit 1 contrôle l'expression des gènes de prolactine et de l'hormone de croissance dans l'hypophyse ; il est également indispensable au développement normal des cellules thyréotropes, lactotropes et somatotropes de cet organe $\left(\mathrm{m} / \mathrm{s}, n^{\circ} 10\right.$, vol. 6, p. 1025). L'interaction entre Pit 1 et son élément cible d'ADN semble, de plus, être responsable du contrôle de l'expression des gènes de prolactine et d'hormone de croissance par l'AMP cyclique, l'EGF (epidermal growth factor) et les esters de phorbol. L'équipe de M.G. Rosenfeld (La Jolla, CA, USA) démontre maintenant que, sous l'action de ces agents, Pit 1 est phosphorylé en deux sites dont l'un (la thréonine 220) est situé dans le domaine Pou-homéo. La phosphorylation de ce site modifie la conformation de Pit 1, augmentant sa dimérisation et modifiant son affinité pour ses cibles d'ADN. Cette modification varie selon l'élément d'ADN considéré, la liaison (et donc très probablement l'activation transcriptionnelle) pouvant être diminuée, peu modifiée ou très augmentée [1]. Voici donc un nouvel exemple de la modulation de l'activité biologique d'un facteur de transcription par modification post-traductionnelle, originale ici en ce que les conséquences en sont diverses selon les éléments d'ADN cibles, et de ce fait selon les gènes contrôlés par Pit. De plus, Pit 1 est un exemple remarquable d'un facteur intervenant, à la fois, dans la différenciation cellulaire, la transcription spécifique de tissu et la réponse à des stimuli externes de nature hormonale. [1. Kapiloff MS, et al. Science 1991 ; 253 : 786-9.]
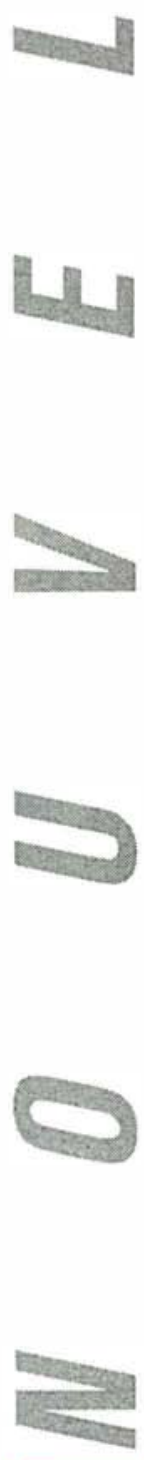